\title{
Concentraciones Séricas de Fenobarbital y Metabolismo de Calcio y Fósforo en el Niño.
}

\author{
Q.F. Teresa Salazar C. ${ }^{1}$; Dr. Francisco Barrera Q. ${ }^{2}$; Nutr. María Teresa Capurro S. ${ }^{1}$; \\ Dr. Cristián Barra B. ${ }^{2}$; Q.F. Alfonso Salinas T. ${ }^{1}$; Dr. Fernando Novoa S. ${ }^{3}$.
}

\section{Serum Phenobarbital Levels and Altered Phospho-Calcic Metabolism.}

Some risk factors have been found between anticonvulsivant therapy and phosphocalcic metabolism alterations. Forty eight epileptic patients, aged 2 to 7 years, treated with phenobarbital, were studied, in comparison with twenty nine healthy controls. Patients with the higher plasma levels of phenobarbital presented the greatest biochemical alterations in the phosphocalcic metabolism. This was specially evident through the increase of alkaline phosphatase activity.

Durante los últimos años ha habido creciente evidencia acerca de los efectos adversos a que están expuestos los pacientes que reciben tratamiento anticonvulsivante. El fenobarbital, debido a su gran uso, ha sido uno de los más ampliamente estudiados. ${ }^{2}-4-6-12$

Entre los efectos adversos del fenobarbital con cierta frecuencia se cita los trastomos en el metabolismo de la vitamina $\mathrm{D}$, también descritos con el uso de difenil hidantoina ${ }^{15-20}$.

En diversos estudios de pacientes adultos $y$ niños, en tratamiento con fenobarbital, primidona o fenitoina, se han observado cambios bioquímicos, radiológicos y clínicos sugerentes de trastomos en el metabolismo de Calcio y Fósforo.

El presente trabajo intenta establecer una relación entre las concentraciones plasmáticas de fenobarbital y alteraciones bioquímicas del metabolismo fosfocálcico.

\section{MATERIAL Y METODO}

Para el estudio se seleccionaron 2 grupos de niños:

48 niños de 2 a 7 años de edad, eutróficos, de ambos sexos, en tratamiento con fenobarbital oral por un período minimo de 6 meses hasta la fecha del estudio, cuya única enfermedad demostrada fue la presencia de sindrome convulsivo. Estos pacjentes son controlados ambulatoriamente en el Servicio de Neurosiquiatria Infantil de] Hospital Paula Jaraquemada. De ellos, 29 correspondían a convulsión febril y 19 a epilepsia.

29 niños de 2 a 7 años de edad, eutróficos, de

1 Centio de Investigaciones Clínicas, Facultad de Medicina, Universidad de Chile.

2 Servicio de Pediatría, Hospital Paula Jaraquemada y Departamento de Pediatría Central División Ciencias Médicas Sur. Facultad de Medicina. Universidad de Chile.

3 Servicio de Neuropsiquiatría Infantil, Hospital Paula Jaraquemada. ambos sexos sin antecedentes de terapia anticonvulsivante $e$ ingesta regular de ningún otro medicamento, que asistían a control de niño sano en el Consultorio de Maipú.

Los nifros de ambas series que cumplian con los requisitos establecidos fueron evaluados me. diante examen físico y entrevista pediatrica, con el fin de descartar antecedentes familiares, perinatales u otras patologias (enfermedades digestivas, endocrinas, metabólicas, genéticas, etc.), que interfirieran con los parámetros bioquímicos del presente estudio.

Se descartaron los niños con antecedente de bajo peso de nacimiento o con actividad física anormal (sedentarios, hiperkinéticos).

En ambos grupos se realizó una evaluación del estado nutricional con antropometría y encuesta alimentaria por tendencia de consumo cuantifi cada, calificándolos según las tablas de peso edad de $M$. Sempé ${ }^{7-8 \cdot 19}$. En ambas series se tomaron 5 cc. de sangre para mediciones de Calcio, Fósforo, Fosfatasas alcalinas, Proteinas totales con índice $\mathrm{A} / \mathrm{G}$ y Aminoácidos. En el grupo con terapia anticonvulsivante se determinaron además las concentraciones séricas de fenobarbital.

Las muestras de sangre fueron procesadas en el Centro de Investigaciones Clínicas de la Universidad de Chile.

Las mediciones de Calcemia, Fosfemia, Fosfatasas alcalinas, Proteínas totales y Albúmina se hicieron por micrométodos colorimétricos ${ }^{3}$. Los aminoácidos se analizaron por cromatografía en capa fina de celulosa microcristalina.

El fenobarbital se midió por radioinmunoanálisis, usando juegos de Clinical Asays (Division of Travenol Laboratories, inc., Cambridge, Massachusetts 02139).

En el presente trabajo se consideraron como valores normales aquellos obtenidos en el grupo control.

\section{RESULTADOS}

Las principales características de ambos 
grupos se reseffan en la Tabla 1, su análisis demuestra que son comparables, diferenciándose solamente en la existencia de Síndrome Convul. sivo $y$ terapia anticonvulsivante, como únicos factores de riesgo de alteración del metabolismo del Calcio y Fósforo. El estudio se planté en niños de 2 a 7 años porque entre ambas edades no se producen cambios importantes en la Fos. femia ni en la actividad de Fosfatasas alcalinas en el suero.

Tabla 1.

Caracter isticas Generales de Ambos Grupos

\begin{tabular}{|c|c|c|c|}
\hline & & Grupe Control & Grupa Fetnobarbita \\
\hline$N^{0}$ Cesos & & 29 & 48 \\
\hline SEXO & $\begin{array}{l}\text { Masculino } \\
\text { Fernenino }\end{array}$ & $\begin{array}{r}20 \\
9\end{array}$ & $\begin{array}{l}22 \\
26\end{array}$ \\
\hline EDAD & $\begin{array}{l}\text { Rento } \\
\text { Promedio }\end{array}$ & $\begin{array}{c}2-7 y \\
4 y 10 \text { meses }\end{array}$ & $\begin{array}{c}2-7 y \\
46 \text { meses }\end{array}$ \\
\hline \multirow{2}{*}{$\begin{array}{l}\text { Tiempo dt } \\
\text { tratamienlo }\end{array}$} & $\begin{array}{l}\text { Duración de } \\
\text { Trataniento }\end{array}$ & $\ldots \ldots$ & $6 \mathrm{~ms}-5 y 4 \mathrm{~s}$ \\
\hline & Promedia & ----- & 2 y 4 meses \\
\hline \multirow{2}{*}{ Diagnátitito } & $\begin{array}{l}\text { Connulsiones } \\
\text { tebribes }\end{array}$ & ----- & 29 \\
\hline & Epilep sia & ----- & 19 \\
\hline Estado Nutritivo & & Noumal & Norral \\
\hline
\end{tabular}

El estado nutriclonal de ambos gropos fue evaluado de scuerdo a las estándares de M. Sempe.

La Tabla 2 se refiere a los promedios de los parámetros analizados más - menos sus respectivas desviaciones estándar. Se observa que no hubo diferencia signifjcativa en los niveles de Calcio sérico, en cambio la Fosfemia del grupo en tratamiento resultó significativamente más baja que la del grupo control $(p<0.001)$. La actividad de las Fosfatasas alcalinas se encontraba significativamente más alta en el grupo en tratamiento con fenobarbital ( $p<0.0001)$.

En la Tabla 3 se muestra la relación entre dosis.
Tabla 2.

Niveles Séricos de los Parámetros en Estudio en Ambos Grupos

Grupo Cantrol Grupo Fenobarbital

Fenobarbital

(jw/mus)

Calcio (mg/d) Promedio $\pm d s$. Promedio \pm ds.

Fósforo (mg/dl) - -

$22,47 \pm 7,93$

Fosfatasas Alcalinas

(Unidades K.A.)

Proteína total

gstallo

Albúmina (g/dl)

$$
9,07 \pm 0,61
$$

$9,29 \pm 0,50$

$5,00 \pm 0,91$

$* 4,22 \pm 0,49$

$26,01 \pm 5,34$

$* * 33,45 \pm 8,62$

$7,24 \pm 0,53$

$7,23 \pm 0,49$

$4,44 \pm 0,34$

$4,57 \pm 0,42$

Rango terapéutico para Fenobarbital en nuestro

Laboratorio es de 8 - $201 \mathrm{~kg} / \mathrm{ml}$.

* $\mathrm{p}<0,001$

$* * p<0,001$

administrada de fenobarbital en $\mathrm{mg} . x \mathrm{~kg}$. de peso corporal, número de pacientes con alteración de las Fosfatasas alcalinas y magnitud de la alteración. El $31,25 \%$ de los pacientes en trata. miento con fenobarbital presentaba actividad alterada de Fosfatasas alcalinas, siendo más evidente aún en el subgrupo que recibió la mayor dosis de fenobarbital $(53,8 \%)$.

La Tabla 4 muestra la relación entre niveles plasmáticos de fenobarbital y grado de alteración en la actividad de las fosfatasas alcalinas, con el porcentaje creciente de pacientes que la presenta. En el grupo de pacientes con niveles de 10 a 19 mg. $x$ ml. de fenobarbital, podemos observar un $19,04 \%$ de ellos con alteraciones de los niveles de $F$. alcalinas, en el segundo grupo (rango de 20 a $29 \mu \mathrm{g}$. $x$ ml.) un $26,66 \%$, mientras que en el tercer grupo cuyos niveles van desde 30 a $44 \mu \mathrm{g}$. $x$ mil, podemos observar un $58,33 \%$ de niños con actividad aumentada de F. alcalinas, alcanzando valotes de hasta $55,86 \mathrm{Uds}$. K.A.

En la Tabla 5 se observa que no hay relación entre duración de la terapia, después de transcurridos los primeros 6 meses, y los efectos a nivel del metabolismo fosfocálcico.

Tabla 3.

Relación entre Dosis Administrada de Fenobartital $y$ Niveles de Fosfatasas Alcalinas.

$\begin{array}{ccccc}\begin{array}{c}\text { Dosis de } \\ \begin{array}{c}\text { Fenobarbital } \\ \text { (mg/kg/dia) }\end{array}\end{array} & \begin{array}{c}\text { Fosfatasas } \\ \text { Alcalinas } \\ \text { Unidades K.A. }\end{array} & \begin{array}{c}\text { Número de } \\ \text { Pacientes }\end{array} & \begin{array}{c}\text { Pacientes con } \\ \text { alteración en } \\ \text { nivel de fostasas } \\ \text { alcalinas }\end{array} & \begin{array}{c}\text { Porcentaje con } \\ \text { aiteración de Fosfatasas } \\ \text { alcalinas }\end{array} \\ 2,46-3,82 & 20,32-42,85 & 14 & 3 & 21,40 \\ 4,04-4,97 & 18,31-50,18 & 21 & 5 & 23,80 \\ 5,05-6,41 & 24,54-55,86 & 13 & 7 & 53,80\end{array}$

$31,25 \%$ de los pacientes en tratamiento con Fenobarbital, mostró niveles alterados de Fosfatasas alcalinas cuando se compararon con el grupo control. 
Tabla 4.

Relación entre Niveles de Fenobarbital y Fosfatasas Alcalinas.

$\begin{array}{ccccc}\begin{array}{c}\text { Fenobarbital } \\ (\mathrm{fg} / \mathrm{ml})\end{array} & \begin{array}{c}\text { Fostatasas } \\ \text { Alcalinas } \\ \text { Unidades K.A. }\end{array} & \begin{array}{c}\text { Número de } \\ \text { casos }\end{array} & \begin{array}{c}\text { Pacientes con } \\ \text { alteraciones en } \\ \text { Fosfatasas Alcalinas }\end{array} & \begin{array}{c}\text { Porcentaje de } \\ \text { pacientes con Fosfatasas } \\ \text { Alcalinas Alteradas }\end{array} \\ 10-19 & 18,31-43,40 & 21 & 4 & 19,04 \\ 20-29 & 20,87-50,18 & 15 & 4 & 26,66 \\ 30-44 & 24,54-55,86 & 12 & 7 & 58,33\end{array}$

$31,25 \%$ de los pacien tes en tratamiento con Fenobarbital, mostró niveles alterados de Fosfatasas Alcalinas cuando se comparó al grupo control.

Tabla 5.

Relacion entre Tiempo de Tratamiento y Niveles Plasmáticos de Fosfatasas Alcalinas.

\begin{tabular}{|c|c|c|}
\hline $\begin{array}{l}\text { Ticmpo de } \\
\text { Tratamiento }\end{array}$ & $N^{\circ}$ de Nińos & $\begin{array}{l}\text { Niveles Plasmaticos de } \\
\text { losiatasas Altalinas } \\
\text { (Unidades Kine Amstuong) }\end{array}$ \\
\hline $6 \mathrm{~ms}$ - 2 anos & 22 & $20,8 T-49,81$ \\
\hline 2a. 1 ms. -4 años & 14 & $20.32-42,85$ \\
\hline a. $1 \mathrm{~ms} .-5 \mathrm{~d}, 4 \mathrm{~ms}$. & 12 & $18.31-55,86$ \\
\hline
\end{tabular}

No se observa relación entre la duración de la terápia y el nivel de Fosfatasas Alcalinas.

\section{DISCUSION}

Estudios recientes demuestran alteraciones del metabolismo de la vitamina $D$ en pacientes en tratamiento anticonvulsivante. Ellas han sido asociadas por algunos autores con la duración del tratamiento $\mathrm{o}^{4-6}$, aunque otros no han encontrado tal relación, situación que coincide con nuestro estudio.

Por otra parte, de las publicaciones disponibles, no es posible discernir la influencia de otros factores en estos hallazgos, corno dietas carenciales, inactividad o reposo relativo de los pacientes, terapias asociadas, exposición al sol, etc. Tampoco se señalan las concentraciones plasmáticas de los anticonvulsivantes en los individuos que mostraron alteraciones.

Rickens y Rowe en 1970 encontraron una relación entre dosis administrada y grado de alteración del metabolismo fosfocálcico ${ }^{15}$. Sin embargo es bien conocida la variabilidad individual de los niveles sanguíneos en el estado estacionario con determinadas dosis de fenobarbital u otro anti convulsivante. Por esta razón nos ha parecido más adecuado investigar si existe relación entre niveles plasmáticos de fenobarbital, la magnitud de la alteración de la actividad de las
Fosfatasas alcalinas y el número de pacientes que la presenta, pudiendo apreciarse una relación directa significativa. Considerando que la mayoría de los laboratorios aceptan como cifras terapéuticas hasta $40 \mu \mathrm{g} \mathrm{x} \mathrm{ml}$, se estaría aceptando un rango útil que sin lugar a dudas presenta riesgos de toxicidad en el nive] bioquímico. Nuestros estudios sugieren que la concentración terapéutica adecuada con fenobarbital es 20 $\mu \mathrm{g} / \mathrm{ml}^{18}$. En todo caso parece conveniente insistir en la necesidad de controlar los niveles de anticonvulsivantes, especialmente en niños, dado su potencial efecto sobre el crecimiento y desa. rrollo.

El mecanismo a través del cual actuarian los anticonvulsivantes aún se discute, aunque injcialmente se planteó que disminuían la biodisponibilidad del calcitriol o metabolito activo de la vitamina D 1-25 dihidroxicolecalciferol, por la aparición de otros metabolitos polares del colecalciferol, mediada por hidroxilaciones abeIrantes causadas por alteración inđucida de la actividad ribosómica hepática. Ha sido difícil identificar dichos metabolitos polares, incluso publicaciones recientes ${ }^{21}$, han mostrado niveles normales de 1-25 dihidroxicolecalciferol, disminución del 25 hidroxicolecalciferol y $24-25$ dihidroxicolecalciferol, al mismo tiempo que aumento de los niveles de Hormona paratiroídea y Fosfatasas alcalinas. La administración diaria de 2.000 Uds. de vitamina $D$ durante 3 semanas, mejora los niveles séricos de 25 hidroxicolecalciferol, aunque no corrige el hiperparatiroidismo secundario ${ }^{2}$.

Como se ha descrito raquitismo bioquímico y radiológico $\mathbf{1 5 . 2 2}^{\mathbf{1 5}}$, se ha recomendado dar a estos pacientes en tratamiento prolongado con anticonvulsivantes, vitamina $\mathrm{D}$ oral, en dosis de 1000 a $2000 \mathrm{Uds} /$ día.

E1 presente trabajo pretende llamar en nuestro medio la atención hacia los efectos colaterales de 
los anticonvulsivantes, dado el elevado número de epilépticos bajo control y tratamiento.

\section{RESUMEN}

Se comparan 48 niños con síndrome convulsivo en tratamiento con fenobarbital y 29 contro. les sanos. Pudo demostrarse que los pacientes con njveles más altos de fenobarbital plasmático presentaron las mayores alteraciones bioquímicas del metabolismo fosfocálcico, hecho especial. mente notorio en el aumento de la actividad de las Fosfatasas alcalinas.

Se ha recomendado suplementar con vitamina $D$ a dichos pacientes.

\section{REFERENCIAS}

1 Battino, D; Cusi, C.: Franceschetti, S.: Moise, A.; Spina, S. and A vanzani, G.: Ethosuximide plasma concentrations: influence of age and associated concomitant therapy. Clin. Phamacokinet. 7: 176-180, 1982.

${ }^{2}$ Buillon, R.; Reynsert, J.; Ches, J.H.; Lisseus, W.; De Moor, P.: The effect of anticonvulsant therapy on serum levels of 25 dihydroxycholecalciferol. J. Clin. Endocrinol. Metab. 41: 1130-1135, 1975.

3 Counerty. Horold V., M.D.; and Briggs, Anglis R., B.S.: Determination of serum calcitm by means of orthocresolphthalein complexone. Am. J. Clin. Pathol. 45: 290-296, 1966.

${ }^{4}$ Crosley, $C$; Chee, $C$; Bermen, P.: Rickets associated with anticonvulsant, Pediatrics 53: 91-95, 1974.

5 De Luca, F.: Vitamin D: Revisited 1980. Clin. Endocrinol. Metab. 9: 3-26, 1980.

${ }^{6}$ Dent, C.E.: Rickens, A.; Rowe, D.J.F.: Osteomalacia with long-term anticonvulsant therapy in Epilepsy. Br. Med. J. 4: 69-72, 1970 .

7 FAO/UNCEP/OMS. Informe de un Comité Mixto de Expertos, Metodología de la vigilancia nutricional. Serie de Informes Técnicos, No 593,1976

${ }^{8}$ Ficha encuesta alimentaria tendencia de consumo cuantificada. Departamento de Nutrición, Facultad de Medicina Norte, Universidad de Chile. Publicación de Investigación, No $67-68,1968$.

9 Hahn, T.J.: Drug induced disorders of vitamin D mineral metabolism. Clin. Endocrinol, Metab. 9: $107-117,1980$.

10 Hawer, E.B.: Clinical implications of measurements of circulating Vitamin D metabolites. Clin. Endocrinol. Metab. 9: 63-79, 1980 .

11 Jubis, W.; Haussler, M.; Mc. Cain, T.A.; Tolman, K.: Plasma 1,25 dihydroxy vitamin $D$ levels in patients receiving anticonvulsivant drugs. $J$, Clin. Endoctinol. Metab. 44; $617-621,1977$.

12 Lester, H.: Rickets associated with anticonvulsant. Pediatrics 53: 91-95, 1974.

13 Mimaki, T.; Walson, P.D.; Hausster, M.R.: Anticonvulsant therapy and Vitamin $D$ metabolism: Evidence for different mechanisms for Phenytoin and Phenobarbital. Pediatric Pharmacol. 1: 105-112, 1980.

${ }^{14}$ Raisz, L.G.: Direct effects of Vitamin D and its metabolites on skeletal tissues. Clin. Endocrinol. Metab. 9: 27-41, 1980.

15 Rickens, A.; Rowe, D.J.F.: Disturbance of calcium metabotism by anticonvulsant drugs. Br. Med. J. 4: $73-76,1970$

${ }^{16}$ Root, A.; Harrison, H.: Recent advances in calcium metabolism. I. Mechanism of calcium homeostasis. J. Pediatr. 88: 1-1B, 1976.

17 Root. A.: Harrison, H.: Recent advances in calcium metabolism. II. Disorders of calcium homeostasis. J. Pediatr. 88: 177-198, 1976.

18 Salazar, T.; Sainas, A.; Novoa, F.: Determination of serum levels in the evaluation of anticonvulsant treatment. Neurocirugía, 35: 159-168, No 3 .

19 Sempé, M.; Roy, M.P.; Pédron, G.: Tabla de crecimiento de peso y talla para niños y nirias de 0 a 14 años de edad. París, 1971.

20 Tolman, K.G.; Jubiz, N.; Saznella, J.J.: Osteomalacia associsted with anticonvulsant drug therapy in mentally retarded children. Pediatrics 56: 45-51, 1975.

21 Keck, E, Gollnick, B, Reinhardt, D.; Karch, D.; Peerenboom, H.; Krüskemper, H. Calcium Metabolism and Vitamin D Metabolite levels in children receiving anticonvulsivants drugs. Eur, J, Pediatr. 139: $52-55,1982$

22 Hurley H. y cols.: Rickets associated with long term anticonvulsivant therapy. Pediatrics 56: 52-55, 1975. 